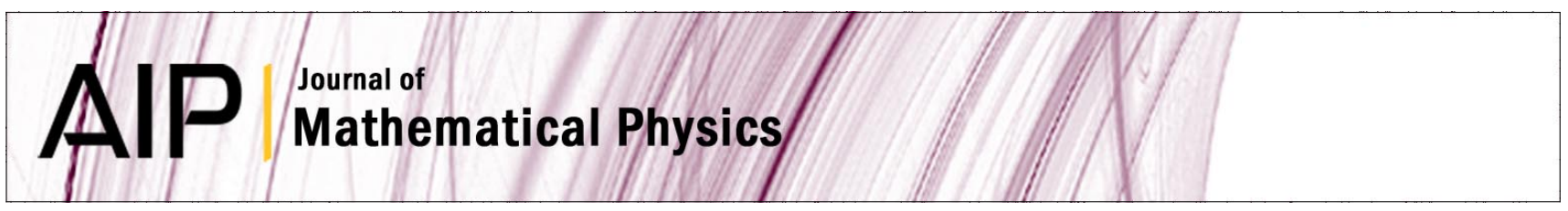

\title{
Deformations of surfaces associated with integrable Gauss-Mainardi-Codazzi equations
}

Ö. Ceyhan, A. S. Fokas, and M. Gürses

Citation: J. Math. Phys. 41, 2251 (2000); doi: 10.1063/1.533237

View online: http://dx.doi.org/10.1063/1.533237

View Table of Contents: http://jmp.aip.org/resource/1/JMAPAQ/v41/i4

Published by the American Institute of Physics.

\section{Additional information on J. Math. Phys.}

Journal Homepage: http://jmp.aip.org/

Journal Information: http://jmp.aip.org/about/about_the_journal

Top downloads: http://jmp.aip.org/features/most_downloaded

Information for Authors: http://jmp.aip.org/authors

\section{ADVERTISEMENT}

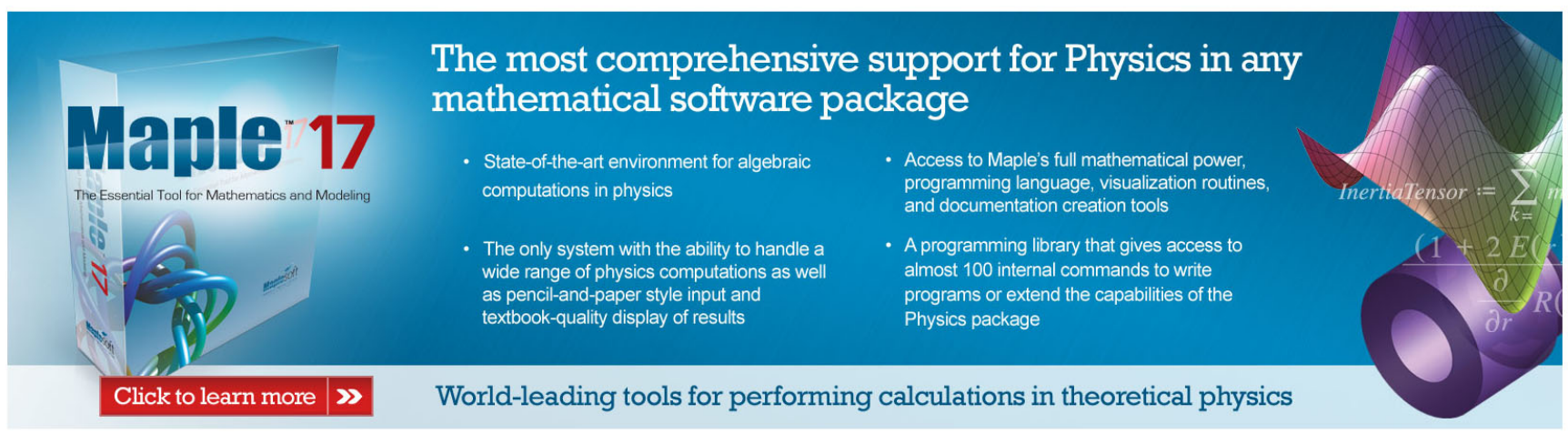




\title{
Deformations of surfaces associated with integrable Gauss-Mainardi-Codazzi equations
}

\author{
Ö. Ceyhan ${ }^{\text {a) }}$ \\ Department of Mathematics, Bilkent University, 06533 Ankara, Turkey
}

A. S. Fokas ${ }^{\text {b) }}$

Institute of Nonlinear Sciences, Clarkson University, Potsdam, Clarkson, New York 13676

M. Gürses ${ }^{\mathrm{c})}$

Department of Mathematics, Bilkent University, 06533 Ankara, Turkey

(Received 6 July 1999; accepted for publication 23 November 1999)

Using the formulation of the immersion of a two-dimensional surface into the three-dimensional Euclidean space proposed recently, a mapping from each symmetry of integrable equations to surfaces in $\mathbb{R}^{3}$ can be established. We show that among these surfaces the sphere plays a unique role. Indeed, under the rigid SU(2) rotations all integrable equations are mapped to a sphere. Furthermore we prove that all compact surfaces generated by the infinitely many generalized symmetries of the sine-Gordon equation are homeomorphic to a sphere. We also find some new Weingarten surfaces arising from the deformations of the modified Kurteweg-de Vries and of the nonlinear Schrödinger equations. Surfaces can also be associated with the motion of curves. We study curve motions on a sphere and we identify a new integrable equation characterizing such a motion for a particular choice of the curve velocity. (C) 2000 American Institute of Physics. [S0022-2488(00)02104-6]

\section{INTRODUCTION}

Let $F: \Omega \rightarrow \mathbb{R}^{3}$ be an immersion of a domain $\Omega \in \mathbb{R}^{2}$ into $\mathbb{R}^{3}$. Let $(u, v) \in \Omega$. The surface $F(u, v)$ is uniquely defined to within rigid motions by the first and second fundamental forms. Let $N(u, v)$ be the normal vector field defined at each point of the surface $F(u, v)$. Then the triple $\left\{F_{u}, F_{v}, N\right\}$ defines a basis of $\mathbb{R}^{3}$ on $S$ parametrized by $F(u, v)$. The motion of this basis on $S$ is characterized by the Gauss-Weingarten $(\mathrm{GW})$ equations. The compatibility of these equations are the well-known Gauss-Mainardi-Codazzi (GMC) equations. The GMC equations are coupled nonlinear partial differential equations for the coefficients $g_{i j}(u, v)$ and $d_{i j}(u, v)$ of the first and second fundamental forms. For certain particular surfaces these equations reduce to a single or to a system of integrable equations. The correspondence between the GMC equations and the integrable equations has been studied extensively, see, e.g., Refs. 1-28.

Recently a more systematic approach to surfaces, GMC equations, and integrable equations has been established by defining surfaces on Lie algebras and on their Lie Groups. ${ }^{1,2}$ In particular this approach provides an explicit relation between symmetries of integrable equations and surfaces in $\mathbb{R}^{3}$. Let the $\mathrm{SU}(2)$ valued function $\Phi(u, v, \lambda)$ satisfy the Lax pair associated with some nonlinear integrable equation for the scalar function $\theta$ (see Refs. 29-32). Define the su(2) valued function $F(u, v, \lambda)$ by

$$
F(u, v, \lambda)=\Phi^{-1}\left(\alpha(\lambda) \frac{\partial \Phi}{\partial \lambda}+M(u, v, \lambda) \Phi+\Phi^{\prime}(\phi)\right),
$$

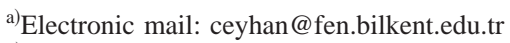

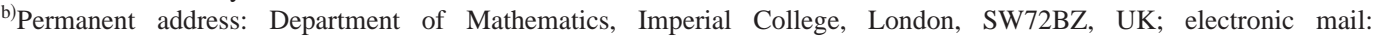
a.fokas@ic.ac.uk

c)Electronic mail: gurses@fen.bilkent.edu.tr
} 
where $\alpha(\lambda)$ is an arbitrary function of the complex constant $\lambda, M(u, v, \lambda)$ is an arbitrary $\operatorname{su}(2)$ valued function of $(u, v), \phi(u, v)$ is a symmetry of the nonlinear equation satisfied by $\theta(u, v)$, and $\Phi^{\prime}$ denotes the Frechét derivative of $\Phi$ with respect to $\theta$. Then $F(u, v, \lambda)$ is the immersion function of a surface $\left(x_{1}, x_{2}, x_{3}\right)$, in $\mathbb{R}^{3}$,

$$
x_{i}=f_{i}(u, v, \lambda), \quad i=1,2,3, \quad F(u, v, \lambda)=i \Sigma_{i=1}^{i=3} f_{i}(u, v, \lambda) \sigma_{i},
$$

where $\sigma_{i}, i=1,2,3$, are the Pauli sigma matrices.

The investigation of some of the consequences of Eq. (1) is the main subject of this paper.

In Sec. II we give a short review of some of the results of Refs. 1 and 2 and also show that if $\alpha=\phi=0$ and $M$ is a constant su(2) matrix, then the surface with immersion function $F$ is a sphere.

In Sec. III we investigate the case that $\theta$ satisfies the sine-Gordon equation

$$
\frac{\partial^{2} \theta}{\partial u \partial v}=\sin \theta
$$

In particular we show the following. (a) If $\alpha=M=0$ and $F$ describes an oriented, compact connected surface, then this surface is homeomorphic to a sphere. This result gives another example to the studies of the global properties of the associated surfaces. ${ }^{16-19,33-37}$ (b) If $\phi=0$ and $M=(i p / 2) \sigma_{1}$, where $p$ is a constant, then $F$ describes a surface of constant negative curvature.

In Sec. IV we investigate the case where $\theta$ satisfies either the elliptic sinh-Gordon or

$$
\frac{\partial^{2} \theta}{\partial u^{2}}+\frac{\partial^{2} \theta}{\partial v^{2}}+\frac{1}{4}\left(H_{0}^{2} e^{2 \theta}-e^{-2 \theta}\right)=0,
$$

or the Liouville equation. In particular we show that special cases of Eq. (1) can be used to generate linear Weingarten surfaces.

In Secs. V and VI we use Eq. (1) and Lax pairs associated with the nonlinear Schrödinger and with the modified Korteweg-de Vries (KdV) equations to characterize certain nonlinear Weingarten surfaces including

$$
\begin{gathered}
2 \mu^{2} H^{2}\left(\mu^{2} K-\nu\right)=\left(3 \mu^{2} K+4 \lambda^{2}-2 \nu\right)^{2}, \\
K-\frac{2}{9} H^{2}+\frac{4 \lambda^{2}}{9 \mu^{2}}=0,
\end{gathered}
$$

where $K$ and $H$ denote the Gaussian and mean curvatures, respectively, and $\mu, \lambda, \nu$ are constants.

Surfaces can also be constructed from the motion of curves, see Appendices A and B. In Sec. VII we study curve motions on a sphere. By choosing a particular velocity vector, we obtain the new integrable equation

$$
\theta_{t}-\theta_{u} \cos \theta \partial_{u}^{-1}\left(\frac{\sin \theta}{(\cos \theta)^{2}} \theta_{t}\right)+\frac{1}{2}\left(\theta_{u} \cos \theta\right)^{3}+\cos \theta\left[\cos \theta\left(\theta_{u} \cos \theta\right)_{u}\right]_{u}=0
$$

Equation (7) reduces to the modified $\mathrm{KdV}$ equation in the limit that the curvature of the curve approaches a constant.

In Sec. VIII we give explicit formulas which associate a curve evolution to a given surface.

\section{SURFACES OF INTEGRABLE EQUATIONS}

In this section we follow the notations of Refs. 1 and 2.

Theorem 2.1: (Ref. 1) Let $U(u, v ; \lambda), V(u, v ; \lambda), A(u, v ; \lambda), B(u, v ; \lambda)$ be $\operatorname{su}(2)$ valued differentiable functions of $u, v$ for $(u, v) \in \Omega \subset \mathbb{R}^{2}$ and $\lambda \in \mathbb{C}$. Assume that these functions satisfy

$$
U_{v}-V_{u}+[U, V]=0,
$$


and

$$
A_{v}-B_{u}+[A, V]+[U, B]=0 .
$$

Define an $\mathrm{SU}(2)$ valued function $\Phi(u, v ; \lambda)$ and an su(2) valued function $F(u, v ; \lambda)$ by

$$
\Phi_{u}=U \Phi, \quad \Phi_{v}=V \Phi,
$$

and

$$
F_{u}=\Phi^{-1} A \Phi, \quad F_{v}=\Phi^{-1} B \Phi .
$$

Then for each $\lambda, F(u, v ; \lambda)$ defines a two-dimensional surface in $\mathbb{R}^{3}$,

$$
x_{j}=F_{j}(u, v ; \lambda), \quad j=1,2,3, \quad F=i \sum_{k=1}^{3} F_{k} \sigma_{k},
$$

where $\sigma_{k}$ are the usual Pauli matrices

$$
\sigma_{1}=\left(\begin{array}{ll}
0 & 1 \\
1 & 0
\end{array}\right), \quad \sigma_{2}=\left(\begin{array}{cc}
0 & -i \\
i & 0
\end{array}\right), \quad \sigma_{3}=\left(\begin{array}{cc}
1 & 0 \\
0 & -1
\end{array}\right) .
$$

The first and second fundamental forms of $S$ are

$$
\begin{gathered}
\left(d s_{\mathrm{I}}\right)^{2}=\langle A, A\rangle d u^{2}+2\langle A, B\rangle d u d v+\langle B, B\rangle d v^{2}, \\
\left(d s_{\mathrm{II}}\right)^{2}=\left\langle A_{u}+[A, U], C\right\rangle d u^{2}+2\left\langle A_{v}+[A, V], C\right\rangle d u d v+\left\langle B_{v}+[B, V], C\right\rangle d v^{2},
\end{gathered}
$$

where

$$
\langle A, B\rangle=-\frac{1}{2} \operatorname{trace}(A B), \quad|A|=\sqrt{\langle A, A\rangle},
$$

and

$$
C=\frac{[A, B]}{|[A, B]|} .
$$

A frame on this surface $S$, is

$$
\Phi^{-1} A \Phi, \quad \Phi^{-1} B \Phi, \quad \Phi^{-1} C \Phi .
$$

The Gauss and mean curvatures of $S$ are given by

$$
K=\operatorname{det}(G), \quad H=\operatorname{trace}(G), \quad G=\left(\begin{array}{ll}
d_{11} & d_{12} \\
d_{12} & d_{22}
\end{array}\right)\left(\begin{array}{ll}
g_{11} & g_{12} \\
g_{12} & g_{22}
\end{array}\right)^{-1} .
$$

The following theorem gives an explicit construction of functions $A, B$ and of the immersion function $F$ from the symmetries of Eqs. (8) and (10):

Theorem 2.2: (Ref. 2) Suppose that $U(u, v)$ and $V(u, v)$ can be parametrized in terms of $\lambda$ and of the scalar function $\theta(u, v)$ in such a way that Eq. (8) is equivalent to a single PDE for $\theta(u, v)$ independent of $\lambda$. This equation, which by definition is called integrable PDE, possesses the Lax pair defined by Eq. (10). Define the su(2) valued functions $A(u, v, \lambda)$ and $B(u, v, \lambda)$ by

$$
A=\alpha \frac{\partial U}{\partial \lambda}+\frac{\partial M}{\partial u}+[M, U]+U^{\prime} \phi,
$$




$$
B=\alpha \frac{\partial V}{\partial \lambda}+\frac{\partial M}{\partial v}+[M, V]+V^{\prime} \phi
$$

where $\alpha(\lambda)$ is an arbitrary scalar function of $\lambda, M(u, v ; \lambda)$ is an su(2) valued arbitrary function of $u, v, \lambda$, the scalar $\phi$ is a symmetry of the partial differential equation (PDE) satisfied by the function $\theta(u, v)$, and the prime denotes Fréchet differentiation. Then there exists a surface with immersion $F(u, v ; \lambda)$ defined in terms of $A, B$ and $\Phi$ by Eqs. (20) and (21). Furthermore, $F$ to within an additive constant, is given by

$$
F=\Phi^{-1}\left(\alpha \frac{\partial \Phi}{\partial \lambda}+M \Phi+\Phi^{\prime} \phi\right) .
$$

Example: Let

$$
M=f_{1} U+f_{2} V+M_{0},
$$

where $M_{0}$ is an $\operatorname{su}(2)$ valued constant matrix and $\alpha(\lambda), f_{1}(\lambda), f_{2}(\lambda)$ are scalar functions of the arguments indicated. Then Eqs. (20)-(21) and (22) become

$$
\begin{gathered}
A=\alpha(\lambda) \frac{\partial U}{\partial \lambda}+\frac{\partial f_{1}}{\partial u} U+f_{1} \frac{\partial U}{\partial u}+\frac{\partial f_{2}}{\partial u} V+f_{2} \frac{\partial U}{\partial v}+f_{3}\left[M_{0}, U\right]+U^{\prime} \phi \\
B=\alpha(\lambda) \frac{\partial V}{\partial \lambda}+\frac{\partial f_{1}}{\partial v} U+f_{1} \frac{\partial V}{\partial u}+\frac{\partial f_{2}}{\partial v} V+f_{2} \frac{\partial V}{\partial v}+f_{3}\left[M_{0}, V\right]+V^{\prime} \phi \\
F=\Phi^{-1}\left(\alpha \frac{\partial \Phi}{\partial \lambda}+f_{1} \partial_{u} \Phi+f_{2} \partial_{v} \Phi+M_{0} \Phi+\Phi^{\prime} \phi\right) .
\end{gathered}
$$

We now study the surfaces generated by constant matrix $M_{0}$ which corresponds to constant $\mathrm{SU}(2)$ rotations of $\Phi$.

Theorem 2.3: Let $A=\left[M_{0}, U\right]$ and $B=\left[M_{0}, V\right]$, where $M_{0}$ is an su(2) constant matrix. Then $K=1 /\left|M_{0}\right|^{2}$ and $H=-2 \epsilon /\left|M_{0}\right|$, where $\epsilon= \pm 1$ and $\left|M_{0}\right|=\sqrt{\left\langle M_{0}, M_{0}\right\rangle}$. Hence all such deformed surfaces are spheres with radii $\left|M_{0}\right|$.

Proof: It is easy to prove that

$$
[A, B]=\alpha M_{0},
$$

where $\alpha$ is the scalar defined by $\alpha=\vec{m} \cdot(\vec{u} \times \vec{v})$. Here $\vec{m}, \vec{u}$, and $\vec{v}$ are the corresponding threevectors of the matrices $M_{0}=(i / 2) \sum_{j=1}^{3} m_{j} \sigma_{j}, U=(i / 2) \sum_{j=1}^{3} u_{j} \sigma_{j}, V=-(i / 2) \sum_{j=1}^{3} v_{j} \sigma_{j}$. Letting $\epsilon=\alpha /|\alpha|$, we find

$$
C=\frac{\epsilon}{\left|M_{0}\right|} M_{0},
$$

hence

$$
\left\langle A_{u}, C\right\rangle=\left\langle A_{v}, C\right\rangle=\left\langle B_{v}, C\right\rangle=0 .
$$

Using these equations it follows that

$$
d_{i j}=-\frac{\epsilon}{\left|M_{0}\right|} g_{i j} \text {. }
$$

Hence $d g^{-1}=\left(-\epsilon /\left|M_{0}\right|\right) I$, where $I$ is the identity matrix. Hence 


$$
\begin{aligned}
& K=\operatorname{det}\left(d g^{-1}\right)=\frac{1}{\left|M_{0}\right|^{2}}, \\
& H=\operatorname{tr}\left(d g^{-1}\right)=-\frac{2 \epsilon}{\left|M_{0}\right|} .
\end{aligned}
$$

QED

This theorem implies that the rigid SU(2) rotations define a map from all integrable equations to the surface of the sphere with a parametrization $F$ such that the coefficients of the first fundamental form takes the form

$$
g_{i j}=\frac{1}{4}\left[m^{2} \vec{u}_{i} \cdot \vec{u}_{j}-\left(\vec{m} \cdot \vec{u}_{i}\right)\left(\vec{m} \cdot \vec{u}_{j}\right)\right],
$$

where $m^{2}=\vec{m} \cdot \vec{m}$, and $\vec{u}_{i}=(\vec{u}, \vec{v})$. The immersion function is given by $F=\Phi^{-1} M_{0} \Phi$.

\section{DEFORMATION OF SINE-GORDON SURFACES}

Consider the motion of the curve with curvature $\rho=\theta_{u}$ and constant torsion $\tau=\lambda$. It is shown in example B.1 that if the velocity of this curve is given by $(0,-(1 / \lambda) \sin \theta,(1 / \lambda) \cos \theta)$, the motion of this curve is characterized by the sine-Gordon equation

$$
\frac{\partial^{2} \theta}{\partial u \partial v}=\sin \theta,
$$

where $\theta(u, v)$ is a real scalar function and time is denoted by $v$. Define $U(u, v, \lambda)$, and $V(u, v, \lambda)$ by

$$
U=\frac{i}{2}\left(-\theta_{u} \sigma_{1}+\lambda \sigma_{3}\right), \quad V=\frac{i}{2 \lambda}\left(\sin \theta \sigma_{2}-\cos \theta \sigma_{3}\right)
$$

Let $\varphi$ be a symmetry of Eq. (33), i.e., let $\varphi$ be a solution of

$$
\frac{\partial^{2} \varphi}{\partial u \partial v}=\varphi \cos \theta
$$

Solutions of (35) contain the geometrical and generalized symmetries of the sine-Gordon equation. $^{38,39}$ Then for each $\varphi$, theorem 2.2 (with $\alpha=0, M=0$ ) implies the surface constructed from

$$
A=-\frac{i}{2} \frac{\partial \varphi}{\partial u} \sigma_{1}, \quad B=\frac{i}{2 \lambda} \varphi\left(\cos \theta \sigma_{2}+\sin \theta \sigma_{3}\right)
$$

where the immersion function is given by $F=\Phi^{-1} \Phi^{\prime}(\varphi)$. Equation (33) is an integrable equation and hence it admits infinitely many symmetries usually referred to as generalized symmetries. Indeed, there exist infinitely many explicit solutions of Eq. (35) in terms of $\theta$ and its derivatives. The first few are

$$
\theta_{u}, \theta_{v}, \theta_{u u u}+\frac{\theta_{u}^{3}}{2}, \theta_{v v v}+\frac{\theta_{v}^{3}}{2}, \ldots
$$

We now study the surfaces corresponding to these generalized symmetries.

Lemma 3.1: Let $S$ be the surface generated by a generalized symmetry of the sine-Gordon equation. That is, let $S$ be the surface generated by $U, V, A, B$ defined by Eqs. (34)-(36). The first and second fundamental forms, the Gaussian, and the mean curvatures of this surface are given by 


$$
\begin{gathered}
d s_{\mathrm{I}}^{2}=\frac{1}{4}\left(\varphi_{u}^{2} d u^{2}+\frac{1}{\lambda^{2}} \varphi^{2} d v^{2}\right), \quad d s_{\mathrm{II}}^{2}=\frac{1}{2}\left(\lambda \varphi_{u} \sin \theta d u^{2}+\frac{1}{\lambda} \varphi \theta_{v} d v^{2}\right), \\
K=\frac{4 \lambda^{2} \theta_{v} \sin \theta}{\varphi \varphi_{u}}, \quad H=\frac{2 \lambda\left(\varphi_{u} \theta_{v}+\varphi \sin \theta\right)}{\varphi \varphi_{u}}
\end{gathered}
$$

An immediate corollary of the above lemma is:

Corollary 3.2: Let $S$ be the particular surface defined in Lemma 3.1 corresponding to $\varphi$ $=\theta_{v}$. This surface is the sphere with

$$
\begin{gathered}
d s_{\mathrm{I}}^{2}=\frac{1}{4}\left(\sin ^{2} \theta d u^{2}+\frac{\theta_{v}^{2}}{\lambda^{2}} d v^{2}\right), \quad d s_{\mathrm{II}}^{2}=\frac{\lambda}{2}\left(\sin ^{2} \theta d u^{2}+\frac{\theta_{v}^{2}}{\lambda^{2}} d v^{2}\right), \\
K=4 \lambda^{2}, \quad H=4 \lambda .
\end{gathered}
$$

Let $S$ be a surface generated by the symmetries of the sine-Gordon equation and defined by the mapping $F: \Omega \rightarrow \mathbb{R}^{3}$. Here $\Omega \subset \mathbb{R}^{2}$ is defined by the regular solutions of the sine-Gordon equation (33). We now present a global result regarding such surfaces.

Theorem 3.3: Let $S$ be a regular surface defined in lemma (3.1) in terms of a generalized symmetry of the sine-Gordon equation. If $S$ is an oriented, compact, and a connected surface then it is homeomorphic to a sphere.

Proof: All compact connected surfaces with the same Euler-Poincare character are homeomorphic. ${ }^{40}$ For compact surfaces the Euler-Poincare character $\chi$ is given by

$$
\chi=\frac{1}{2 \pi} \int_{\Omega} \int \sqrt{\operatorname{det}(g)} K d u d v
$$

Since $g=\operatorname{det} g_{i j}=\varphi^{2} \varphi_{u}^{2} / \lambda^{2}, i, j=1,2$, then the integrand $\sqrt{g} K$ in (42) simply becomes

$$
\sqrt{g} K=\lambda \theta_{v} \sin \theta \text {. }
$$

Hence $\chi$ is independent of the deformations $\varphi$, i.e.,

$$
\chi=\frac{\lambda}{2 \pi} \int_{\Omega} \int \theta_{v} \sin \theta d u d v
$$

This proves that $\chi$ has the same value for all generalized symmetries and hence for all sine-Gordon deformed surfaces. Thus in order to calculate $\chi$ it is enough to choose the simplest case. According to Corollary 3.2 the choice $\varphi=\theta_{v}$ leads to a sphere with radius $1 / 2 \lambda$, where $\chi=2$. Hence all deformed surfaces have the Euler-Poincare character $\chi=2$. Therefore they are all homeomorphic to a sphere. This completes the proof of the theorem.

QED

Compact connected surfaces with $K>0$ are called ovaloids. They all have $\chi=2$. Hence we have a corollary to theorem 3.3 concerning such surfaces.

Corollary 3.4: Surfaces defined in Theorem 3.3 are also homeomorphic to ovaloids.

Solitonic solutions of the sine-Gordon equation satisfy the rapidly decaying conditions, $\theta( \pm \infty)=0, \theta_{u}( \pm \infty)=0, \theta_{v}( \pm \infty)=0, \ldots$. Then for such a case we have the following lemma.

Lemma 3.5: Let $S$ be the surface defined in Lemma (3.1). Suppose that this surface is noncompact. If the associated solution $\theta(u, v)$ of the sine-Gordon equation satisfies the conditions that $\theta, \theta_{u}, \theta_{v}, \ldots$ tend to zero as $u \rightarrow \pm \infty$, then

$$
\int_{-\infty}^{\infty} \sqrt{\operatorname{det}(g)} K d u=0
$$


We now consider a different class of surfaces which are also constructed from solutions of the sine-Gordon equation.

Lemma 3.6: Let $S$ be the surface constructed from $U, V$ given by Eq. (27) and from $A$ $=\mu(\partial U / \partial \lambda), B=\mu(\partial V / \partial \lambda)$ where $\mu$ is a scalar depending on $\lambda$. This surface has the following fundamental forms and curvatures:

$$
\begin{gathered}
d s_{\mathrm{I}}^{2}=\frac{\mu^{2}}{4}\left(d u^{2}+\frac{2}{\lambda^{2}} \cos \theta d v d v+\frac{1}{\lambda^{4}} d v^{2}\right), \quad d s_{\mathrm{II}}^{2}= \pm \frac{\mu}{\lambda} \sin \theta d v d v, \\
K=-\frac{4 \lambda^{2}}{\mu^{2}}, \quad H= \pm \frac{4 \lambda}{\mu} \cot (\theta) .
\end{gathered}
$$

Corollary 3.7: Let $\theta$ be a rapidly decaying solution of the sine-Gordon equation and $S$ be the surface defined in Lemma (3.6). Then

$$
\int_{-\infty}^{\infty} \sqrt{\operatorname{det}(g)} K d u=0
$$

Proof: This is a consequence of

$$
\sqrt{\operatorname{det}(g)} K=-\sin \theta=-\theta_{u v} .
$$

QED

We now consider yet a different class of surfaces associated with solutions of the sine-Gordon equation.

Lemma 3.8: Let $S$ be the surface constructed from $U$ and $V$ defined by Eq. (34) and from

$$
A=\mu \frac{\partial U}{\partial \lambda}+\frac{i p}{2}\left[\sigma_{1}, U\right], \quad B=\mu \frac{\partial V}{\partial \lambda}+\frac{i p}{2}\left[\sigma_{1}, V\right],
$$

where $\mu$ and $p$ are scalars depending on $\lambda$. The immersion function $F$ is given by

$$
F=\Phi^{-1}\left[\mu \frac{\partial \Phi}{\partial \lambda}+\frac{i p}{2} \sigma_{1} \Phi\right] .
$$

This surface is parallel to a surface of negative constant curvature. The distance between these surface is $p / 4$.

Proof: A straightforward but lengthy calculation implies that for this surface

$$
\left(\mu^{2}+\lambda^{2} p^{2}\right) K+2 p \lambda^{2} H+4 \lambda^{2}=0 .
$$

Let $K_{0}$ and $H_{0}$ be the Gaussian and mean curvatures of a surface $S_{0}$ with constant curvature $K_{0}$ and let $S$ be parallel to $S_{0}$, then ${ }^{40}$

$$
K_{0}=\frac{K}{1-2 a H+a^{2} K}, \quad H_{0}=\frac{H-a K}{1-2 a H+a^{2} K},
$$

where $a$ is a constant. Hence comparing the first equation in Eq. (50) and (49) we find that

$$
a=\frac{p}{4}, \quad K_{0}=-\frac{16 \lambda^{2}}{3 p^{2}+4 \mu^{2}} .
$$

Hence $S$ is parallel to a surface $S_{0}$ with negative constant curvature. $p / 4$ is the distance between the surfaces.

There exists a particular case where the geometrical quantities become simpler: 
Lemma 3.9: Let $S$ be the surface in Lemma (3.8) with $\mu=\lambda p$. Then

$$
\begin{gathered}
d s_{\mathrm{I}}^{2}=\frac{p^{2}}{2}\left(\lambda^{2} d u^{2}-2 \sin \theta d u d v+\frac{1}{\lambda^{2}} d v^{2}\right), \\
d s_{\mathrm{II}}^{2}=\frac{p}{2}\left[\lambda^{2} d u^{2}-2(\sin \theta+\cos \theta) d u d v+\frac{1}{\lambda^{2}} d v^{2}\right], \\
K=-\frac{2}{p^{2}} \tan \theta, \quad H=\frac{2}{p}-\frac{2}{p} \tan \theta .
\end{gathered}
$$

The curvature density $\sqrt{\operatorname{det}(g)} K$ has a form similar to the one in Corollary 3.7. Thus $\sqrt{\operatorname{det}(g)} K$ $=-\sin \theta=-\theta_{u v}$.

The following corollary of the Lemma (3.9) is for solitonic solutions of the sine-Gordon equation:

Corollary 3.10: Let $\theta$ be a rapidly decaying solution of the sine-Gordon equation and $S$ be the surface defined in Lemma (3.9). Then

$$
\int_{-\infty}^{\infty} \sqrt{\operatorname{det}(g)} K d u=0
$$

\section{SURFACES ASSOCIATED WITH THE SINH-GORDON EQUATION}

The sinh-Gordon equation is defined by

$$
\frac{\partial^{2} \theta}{\partial u^{2}}+\frac{\partial^{2} \theta}{\partial v^{2}}+\frac{1}{4}\left(H_{0}^{2} e^{2 \theta}-e^{-2 \theta}\right)=0,
$$

where $\theta(u, v)$ is a real scalar function and $H_{0} \neq 0$ is a real constant. This equation is usually associated with surfaces of constant mean curvature $H_{0}$. In what follows we will show that this equation can also be used to construct several other classes of interesting surfaces.

Lemma 4.1: Let the real scalar function $\theta(u, v)$ be a solution of the hyperbolic sine-Gordon equation (54), where $H_{0} \neq 0$ is a real constant. Define the $\operatorname{su}(2)$ valued functions $U, V, A, B$ by

$$
\begin{gathered}
U=\frac{i}{4}\left[\cos \lambda\left(H_{0} e^{\theta}+e^{-\theta}\right) \sigma_{1}-\sin \lambda\left(H_{0} e^{\theta}-e^{-\theta}\right) \sigma_{2}+2 \theta_{v} \sigma_{3}\right], \\
V=-\frac{i}{4}\left[\sin \lambda\left(H_{0} e^{\theta}+e^{-\theta}\right) \sigma_{1}+\cos \lambda\left(H_{0} e^{\theta}-e^{-\theta}\right) \sigma_{2}+2 \theta_{u} \sigma_{3}\right], \\
A=2 \mu \frac{\partial U}{\partial \lambda}+\frac{i p}{2}\left[\sigma_{3}, U\right], \quad B=2 \mu \frac{\partial V}{\partial \lambda}+\frac{i p}{2}\left[\sigma_{3}, V\right],
\end{gathered}
$$

where $\mu$ and $p$ are real constants. The immersion function $F$ is given by

$$
F=\Phi^{-1}\left[2 \mu \frac{\partial \Phi}{\partial \lambda}+\frac{i p}{2} \sigma_{3} \Phi\right]
$$

The associated surface $S$ has the following fundamental forms and curvatures:

$$
g_{11}=\frac{1}{16 e^{2 \theta}}\left(\left[e^{2 \theta} H_{0}^{2}(2 \mu+p)+(p-2 \mu)\right]^{2}+4 H_{0}\left(4 \mu^{2}-p^{2}\right) \sin ^{2} \lambda e^{2 \theta}\right),
$$




$$
\begin{gathered}
g_{12}=\frac{H_{0}\left(4 \mu^{2}-p^{2}\right) \sin 2 \lambda}{8}, \\
g_{22}=\frac{1}{16 e^{2 \theta}}\left(\left[e^{2 \theta} H_{0}^{2}(2 \mu+p)-(p-2 \mu)\right]^{2}-4 H_{0}\left(4 \mu^{2}-p^{2}\right) \sin ^{2} \lambda \epsilon^{2 \theta}\right), \\
d_{11}=\frac{-H_{0}^{2} e^{4 \theta}(p+2 \mu)-p+2 \mu-2 p H_{0} \cos 2 \lambda e^{2 \theta}}{8 e^{2 \theta}}, \\
d_{22}=\frac{-H_{0}^{2} e^{4 \theta}(p+2 \mu)-p+2 \mu+2 p H_{0} \cos 2 \lambda e^{2 \theta}}{8 e^{2 \theta}} \\
K=4 \frac{p H_{0} \sin 2 \lambda}{4}, \\
d^{4 \theta} H_{0}^{2}(2 \mu+p)^{2}-(2 \mu-p)^{2}
\end{gathered}
$$

It is easy to show that $K$ and $H$ satisfy the following Weingarten relation:

$$
\left(p^{2}-4 \mu^{2}\right) K+2 p H+4=0 .
$$

There exists some interesting particular limiting cases. If $p= \pm 2 \mu, S$ is a surface of constant mean curvature

$$
\begin{gathered}
p=2 \mu, \quad H=-\frac{1}{\mu}, \quad K=\frac{e^{4 \theta} H_{0}^{2}-1}{4 \mu^{2} H_{0}^{2} e^{4 \theta}}, \\
p=-2 \mu, \quad H=\frac{1}{\mu}, \quad K=-\frac{e^{4 \theta} H_{0}^{2}-1}{4 \mu^{2}} .
\end{gathered}
$$

If $p=0, S$ is a surface of constant Gaussian curvature,

$$
\begin{gathered}
K=\frac{1}{\mu^{2}}, \\
H=-\left(\frac{2}{\mu}\right) \frac{H_{0}^{2} e^{4 \theta}+1}{H_{0}^{2} e^{4 \theta}-1} .
\end{gathered}
$$

If $\mu=0, S$ is a sphere.

Surfaces Associated with the Liouville equation: The Liouville equation can be obtained from the sinh-Gordon equation in the limit $H_{0}=0$,

$$
\frac{\partial^{2} \theta}{\partial u^{2}}+\frac{\partial^{2} \theta}{\partial v^{2}}-\frac{1}{4} e^{-2 \theta}=0 .
$$

Lemma 4.2: Let the real scalar function $\theta(u, v)$ be a solution of the Liouville equation (72). Define $U, V, A, B$ by 


$$
\begin{gathered}
U=\frac{i}{4}\left(e^{-\theta} \cos \lambda \sigma_{1}+e^{-\theta} \sin \lambda \sigma_{2}+2 \theta_{v} \sigma_{3}\right), \\
V=-\frac{i}{4}\left(e^{-\theta} \sin \lambda \sigma_{1}-e^{-\theta} \cos \lambda \sigma_{2}+2 \theta_{u} \sigma_{3}\right),
\end{gathered}
$$

where $A$ and $B$ are given in (57) with $p \neq \pm 2 \mu$. The immersion function $F$ is given in (58) with $H_{0}=0$. Then the associated surface $S$ has the following fundamental forms and curvatures:

$$
\begin{gathered}
d s_{\mathrm{I}}=\frac{1}{16} e^{-2 \theta}(2 \mu-p)^{2}\left(d u^{2}+d v^{2}\right), \\
d s_{\mathrm{II}}=-\frac{1}{8} e^{-2 \theta}(2 \mu-p)\left(d u^{2}+d v^{2}\right), \\
K=\frac{4}{(2 \mu-p)^{2}}, \\
H=-\frac{4}{2 \mu-p} .
\end{gathered}
$$

Thus for any $\mu, p$ with $p \neq 2 \mu, S$ is a sphere.

\section{DEFORMATIONS OF THE NONLINEAR SCHRÖDINGER SURFACES}

The nonlinear Schrödinger (NLS) equation is an equation for a complex function $\theta(u, v)$. Letting $\theta(u, v)=r(u, v)+i s(u, v)$, the real valued functions $r$ and $s$ satisfy

$$
\begin{gathered}
r_{v}=s_{u u}+2 s\left(r^{2}+s^{2}\right), \\
s_{v}=-r_{u u}-2 r\left(r^{2}+s^{2}\right) .
\end{gathered}
$$

The associated $U$ and $V$ matrices defining its Lax pair are given by

$$
\begin{gathered}
U=\frac{i}{2}\left(\begin{array}{cc}
-2 \lambda & 2(s-i r) \\
2(s+i r) & 2 \lambda
\end{array}\right), \\
V=-\frac{i}{2}\left(\begin{array}{cc}
-4 \lambda^{2}+2\left(r^{2}+s^{2}\right) & v_{1}-i v_{2} \\
v_{1}+i v_{2} & 4 \lambda^{2}-2\left(r^{2}+s^{2}\right)
\end{array}\right),
\end{gathered}
$$

where

$$
v_{1}=2 r_{u}+4 \lambda s, \quad v_{2}=-2 s_{u}+4 \lambda r
$$

Lemma 5.1: Let $U$ and $V$ be defined by Eqs. (81) and (82), where $r, s$ satisfy the integrable nonlinear equations (79) and (80) and $v_{1}, v_{2}$ are defined by (83). Let $A, B$ be defined by $A$ $=\mu(\partial U / \partial \lambda), B=\mu(\partial V / \partial \lambda)$, where $\mu$ is a real constant, i.e.,

$$
A=\frac{i}{2}\left(\begin{array}{cc}
-2 \mu & 0 \\
0 & 2 \mu
\end{array}\right), \quad B=-\frac{i}{2}\left(\begin{array}{cc}
-8 \lambda \mu & 4 \mu(s-i r) \\
4 \mu(s+i r) & 8 \lambda \mu
\end{array}\right) .
$$

Let the new variables $q$ and $\phi$ be defined in terms of $r$ and $s$ by

$$
r=q \cos \phi, \quad s=q \sin \phi .
$$

In terms of these variables the NLS equations (79) and (80) become 

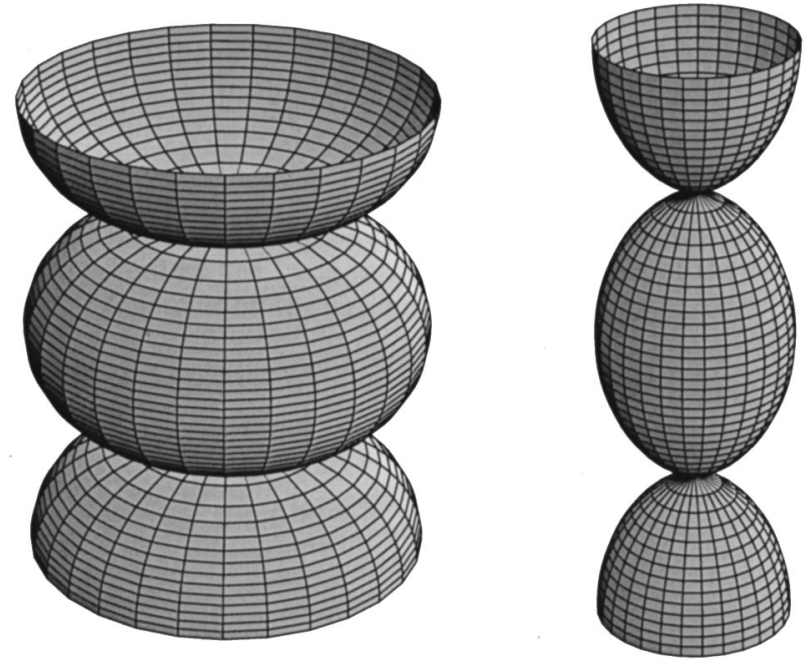

FIG. 1. Weingarten surfaces of the form (94) with $\lambda=0$.

$$
\begin{gathered}
q \phi_{v}=-q_{u u}-2 q^{3}+q \phi_{u}^{2}, \\
q_{v}=q \phi_{u u}+2 q_{u} \phi_{u} .
\end{gathered}
$$

Then the geometrical quantities of the surface $S$ associated with the su(2) valued functions $U, V, A, B$ defined in (81), (82), and (84) can be expressed in terms of the new variables $q$ and $\phi$ through the following equations:

$$
\begin{gathered}
d s_{\mathrm{I}}^{2}=\mu^{2}\left[(d u-4 \lambda d v)^{2}+4 q^{2} d v^{2}\right], \\
d s_{\mathrm{II}}^{2}=-2 \mu q\left[d u-\left(-\phi_{u}+2 \lambda\right) d v\right]^{2}+2 \mu q_{u u} d v^{2}, \\
K=-\frac{q_{u u}}{\mu^{2} q}, \\
H=\frac{q_{u u}-q\left(\phi_{u}+2 \lambda^{2}\right)-4 q^{3}}{2 \mu q^{2}} .
\end{gathered}
$$

The immersion function is given by $F=\Phi^{-1} \mu(\partial \Phi / \partial \lambda)$. In particular if $\phi=\nu v$, where $\nu$ is a real constant, $q=q(u)$, then $q(u)$ satisfies (Fig. 1)

$$
q_{u u}=-2 q^{3}-\nu q
$$

Lemma 5.3: Let $U, V, A, B$ be defined by Eqs. (81), (82), and (84) where $r=q(u) \sin (\nu v), s$ $=q(u) \cos (\nu v), \lambda, \nu, \mu$ are constants and $q(u)$ satisfies $(92)$. Then the associated surface $S$ is a Weingarten surface satisfying the relation

$$
2 \mu^{2} H^{2}\left(\mu^{2} K-\nu\right)=\left(3 \mu^{2} K+4 \lambda^{2}-2 \nu\right)^{2} .
$$

If $\nu=-4 \lambda^{2}$ the above-mentioned Weingarten relation becomes quadratic,

$$
K-\frac{2}{9} H^{2}+\frac{4 \lambda^{2}}{9 \mu^{2}}=0 .
$$




\section{DEFORMATIONS OF THE MODIFIED KORTEWEG-DE VRIES SURFACES}

Let $\rho(u, v)$ satisfy the so-called modified Korteweg-de Vries (mKdV) equation

$$
\rho_{v}=\rho_{u u u}+\frac{3}{2} \rho^{2} \rho_{u} .
$$

The associated $U$ and $V$ matrices defining its Lax pair are given by

$$
\begin{gathered}
U=\frac{i}{2}\left(\begin{array}{cc}
\lambda & -\rho \\
-\rho & -\lambda
\end{array}\right), \\
V=-\frac{i}{2}\left(\begin{array}{cc}
-\frac{\lambda \rho^{2}}{2}+\lambda^{3} & v_{1}-i v_{2} \\
v_{1}+i v_{2} & \frac{\lambda \rho^{2}}{2}-\lambda^{3}
\end{array}\right),
\end{gathered}
$$

where

$$
v_{1}=\rho_{u u}+\frac{\rho^{3}}{2}-\lambda^{2} \rho, \quad v_{2}=-\lambda \rho_{u} .
$$

Lemma 6.1: Let $U$ and $V$ be defined by Eqs. (96) and (97) where the scalar function $\rho(u, v)$ satisfies the $\mathrm{mKdV}$ equation (95) and $v_{1}, v_{2}$ are defined by Eq. (98). Let $A$ and $B$ be defined by $A=\mu(\partial U / \partial \lambda), B=\mu(\partial V / \partial \lambda)$, where $\mu$ is a real constant, i.e., let

$$
\begin{aligned}
& A=\frac{i}{2}\left(\begin{array}{cc}
\mu & 0 \\
0 & -\mu
\end{array}\right) \text {, } \\
& B=-\frac{i}{2}\left(\begin{array}{cc}
\frac{-\mu \rho^{2}}{2}+3 \mu \lambda^{2} & -2 \mu \lambda \rho+i \mu \rho_{u} \\
-2 \mu \lambda \rho-i \mu \rho_{u} & \frac{\mu \rho^{2}}{2}-3 \mu \lambda^{2}
\end{array}\right)
\end{aligned}
$$

The geometrical quantities of the surface $S$ associated with these $U, V, A, B$ are given by

$$
\begin{aligned}
K= & \frac{4 \lambda^{2}}{\mu^{2}\left(\rho_{u}^{2}+4 \lambda^{2} \rho^{2}\right)^{2}}\left[4 \rho^{3} \rho_{u u u u}-4 \rho^{2} \rho_{u} \rho_{u u u}-4 \rho^{2}\left(\rho_{u u}\right)^{2}+4 \rho \rho_{u}^{2} \rho_{u u}-4 \lambda^{2} \rho^{3} \rho_{u u}+4 \rho^{5} \rho_{u u}-\rho_{u}^{4}\right. \\
& \left.+8 \rho^{4} \rho_{u}^{2}\right]
\end{aligned}
$$

$H=\frac{4 \lambda}{\mu\left(\rho_{u}^{2}+4 \lambda^{2} \rho^{2}\right)^{3 / 2}}\left[-\rho \rho_{u u u u}+\rho_{u} \rho_{u u u}-3 \lambda^{2} \rho \rho_{u u}-\rho^{3} \rho_{u u}+2 \lambda^{2} \rho_{u}^{2}-3 \rho^{2} \rho_{u}^{2}-4 \lambda^{4} \rho^{2}-4 \lambda^{2} \rho^{4}\right]$,

$$
d s_{\mathrm{I}}^{2}=\frac{\mu^{2}}{4}\left[\left(d u+\frac{1}{2}\left(\rho^{2}-6 \lambda^{2}\right) d v\right)^{2}+\left(\rho_{u}^{2}+4 \lambda^{2} \rho^{2}\right) d v^{2}\right]
$$

$$
\begin{aligned}
d s_{\mathrm{II}}^{2}= & \frac{\lambda \mu}{\left(\rho_{u}^{2}+4 \lambda^{2} \rho^{2}\right)_{1 / 2}}\left[-\rho^{2} d u^{2}+\left(-2 \rho \rho_{u и}+\rho_{u}^{2}+2 \lambda^{2} \rho^{2}-\rho^{4}\right) d u d v+\frac{1}{4}\left(-4 \rho \rho_{\text {ииии }}+4 \rho_{u} \rho_{\text {ииии }}\right.\right. \\
& \left.+12 \lambda^{2} \rho \rho_{u u}-8 \rho^{3} \rho_{u u}-4 \lambda^{2} \rho_{u}^{2}-6 \rho^{2} \rho_{u}^{2}-4 \lambda^{4} \rho^{2}+4 \lambda^{2} \rho^{4}-\rho^{6}\right) d v^{2}
\end{aligned}
$$


The immersion function is given by $F=\Phi^{-1} \mu(\partial \Phi / \partial \lambda)$. A particular reduction of the abovementioned surface is a Weingarten surface with a complicated Weingarten relation.

Lemma 6.2: Let $U, V$ be defined by Eqs. (96) and (97) where $\lambda, \mu, \alpha$ are constants and $\rho(u)$ satisfies

$$
\rho_{u u}=\alpha \rho-\frac{\rho^{3}}{2} .
$$

Then the associated surface $S$ is a Weingarten surface satisfying the relation

$$
\mu^{2} H^{2} \rho^{2}\left[4\left(\alpha+4 \lambda^{2}\right)-\rho^{2}\right]^{3}=16 \lambda^{2}\left[\rho^{4}-6 \rho^{2}\left(\alpha+4 \lambda^{2}\right)-8 \lambda^{2}\left(\alpha+4 \lambda^{2}\right)\right]^{2},
$$

where

$$
\rho^{2}=4\left(\alpha+4 \lambda^{2}\right)+\frac{16 \lambda^{2}}{\mu} \sqrt{\frac{\alpha+4 \lambda^{2}}{K+4 \lambda^{2} / \mu^{2}}} .
$$

It is interesting that using a different Lax pair for Eq. (105) it is possible to obtain a Weingarten surface simpler than the above:

Lemma 6.3: Let $U, V$ be defined by

$$
V=-\frac{i}{2}\left(\begin{array}{cc}
U=\frac{i}{2}\left(\begin{array}{cc}
\lambda & -\rho \\
-\rho & -\lambda
\end{array}\right), \\
(\alpha+\lambda) \rho+i \rho_{u} & -\frac{\rho^{2}}{2}+\left(\alpha+\alpha \lambda+\lambda^{2}\right)
\end{array}\right),
$$

where $\lambda, \alpha$ are constants and $\rho(u)$ satisfies Eq. (105). Let $A$ and $B$ be defined by $A$ $=\mu(\partial U / \partial \lambda)$ and $A=\mu(\partial V / \partial \lambda)$, where $\mu$ is a constant, i.e., let

$$
\begin{gathered}
A=\frac{i}{2}\left(\begin{array}{cc}
\mu & 0 \\
0 & -\mu
\end{array}\right), \\
B=-\frac{i}{2}\left(\begin{array}{cc}
-(\alpha \mu+2 \mu \lambda) & \mu \rho \\
\mu \rho & \alpha \mu+2 \mu \lambda
\end{array}\right) .
\end{gathered}
$$

The geometrical quantities of the surface $S$ associated with these $U, V, A, B$ are given by

$$
\begin{gathered}
K=\frac{2}{\mu^{2}}\left[\rho^{2}-2 \alpha\right], \quad H=\frac{1}{\mu \rho}\left[3 \rho^{2}+2\left(\lambda^{2}-\alpha\right)\right], \\
d s_{\mathrm{I}}^{2}=\frac{\mu^{2}}{4}\left[(d u+(\alpha+2 \lambda) d v)^{2}+\rho^{2} d v^{2}\right], \\
d s_{\mathrm{II}}^{2}=\frac{\mu \rho}{2}[d u+(\alpha+\lambda) d v]^{2}+\frac{\mu \rho}{4}\left(\rho^{2}-2 \alpha\right) d v^{2} .
\end{gathered}
$$

The immersion function is given by $F=\Phi^{-1} \mu(\partial \Phi / \partial \lambda)$. This surface is a Weingarten surface satisfying the relation

$$
2 \mu^{2} H^{2}\left(\mu^{2} K+4 \alpha\right)=\left[3 \mu^{2} K+4 \lambda^{2}+8 \alpha\right]^{2}
$$


In the special case $\alpha=\lambda^{2}$, this relation becomes

$$
2 \mu^{2} H^{2}=9\left[\mu^{2} K+4 \lambda^{2}\right] .
$$

\section{INTEGRABLE SPHERICAL CURVES}

Consider the motion of a curve on a sphere of radius $1 / \lambda$. Assume that $\rho_{u} \neq 0$. Then, using the results of Proposition A.1 it follows that its motion is characterized by

$$
\theta_{t}=\frac{c_{0}}{\lambda} \cos \theta \theta_{u}+\frac{1}{\lambda} \cos \theta V_{2 u}+\lambda \partial_{u}^{-1}\left(\frac{V_{2}}{\cos \theta}\right)+\theta_{u} \cos \theta \partial_{u}^{-1}\left(\frac{\sin \theta}{(\cos \theta)^{2}} \theta_{t}+\frac{1}{\lambda} V_{2} \theta_{u}\right),
$$

where

$$
\rho=\frac{\lambda}{\cos \theta}, \quad \tau=\theta_{u} .
$$

The velocities $V_{1}$ and $V_{3}$ are given in terms of $V_{2}$ and $\theta$ by

$$
V_{1}=\partial_{u}^{-1}\left(\frac{\lambda \sin \theta}{\cos ^{2} \theta} \theta_{t}+V_{2} \theta_{u}\right)+c_{0}, \quad V_{3}=-\frac{\cos \theta}{\lambda}\left(V_{2 u}+V_{1} \theta_{u}\right),
$$

where $c_{0}$ is an arbitrary constant.

Proof: Spherical curves can be parametrized by (118), since for spherical curves, ${ }^{41}$

$$
\left(\frac{\rho_{u}}{\rho^{2} \tau}\right)^{2}+\frac{1}{\rho^{2}}=\frac{1}{\lambda^{2}}
$$

The last equation in (144) can be written as $V_{3}=-\left(V_{2 u}+V_{1} \tau\right) / \rho$, which is the second equation (119). The first two equations in (144) imply (117) and the first equation of (119).

An integrable motion of a spherical curve. The motion of the curve on a sphere of radius $(1 / \lambda)$ is characterized by Eqs. (117)-(119), where $V_{2}$ is an arbitrary function. Hence each choice of this function yields a spherical surface. Let the velocity component $V_{2}$ of this curve be given by

$$
V_{2}=-\lambda \cos \theta\left(\theta_{u} \cos \theta\right)_{u},
$$

and let $c_{0}=\lambda^{3}$, then $\theta$ evolves according to the integrable equation

$$
\theta_{t}-\theta_{u} \cos \theta \partial_{u}^{-1}\left(\frac{\sin \theta}{(\cos \theta)^{2}} \theta_{t}\right)+\frac{1}{2}\left(\theta_{u} \cos \theta\right)^{3}+\cos \theta\left[\cos \theta\left(\theta_{u} \cos \theta\right)_{u}\right]_{u}=0 .
$$

It seems that Eq. (122) has not appeared before in the soliton literature. We note that in the small $\theta$ limit this equation reduces to the potential modified $\mathrm{KdV}$ equation.

We note that the motion of curves on a sphere was studied recently in Ref. 8 by demanding that the geodesic curvature of these curves is constant and equal to $1 / \lambda$. It can be shown that this requirement is equivalent to $\rho=1 / \lambda$, (i.e., $\rho_{u}=0$ ). Thus the integrable evolutions obtained in Ref. 8 coincide with the modified KdV hierarchy.

\section{CURVES FROM SURFACES}

Appendices A and B show that it is possible to construct surfaces from the motion of curves. It is also possible to associate a curve evolution with a given surface. For this purpose it is more suitable to introduce the Darboux frame on curves. ${ }^{40}$ 
Let $S$ be an oriented regular surface and $\alpha: I \rightarrow S$ be a curve $C$ parametrized by its arc length. At the point $p=\alpha(s)$ consider the following three unit vectors, called the Darboux trihedron: $\mathbf{T}(s)$ is the tangent vector to $C$ at $p, \mathbf{n}(s)$ is the normal vector to $S$ at $p$, and $\mathbf{b}(s)=\mathbf{n}(s)$ $\times \mathbf{T}(s)$. These vectors satisfy the Darboux equations

$$
\begin{gathered}
\frac{d \mathbf{T}}{d s}=\rho_{g} \mathbf{b}+\rho_{n} \mathbf{n}, \\
\frac{d \mathbf{b}}{d s}=-\rho_{g} \mathbf{T}+\tau_{g} \mathbf{n}, \\
\frac{d \mathbf{n}}{d s}=-\rho_{n} \mathbf{T}-\tau_{g} \mathbf{b},
\end{gathered}
$$

where $\rho_{g}=\rho_{g}(s), \rho_{n}=\rho_{n}(s), \tau_{g}=\tau_{g}(s), s \in I$. The geometrical meaning of these coefficients is the following: The scalar $\tau_{g}=-d \mathbf{N} / d s \cdot \mathbf{b}$ is called the geodesic torsion of the curve $C$. This curve is a line of curvature of $S$ if and only if $\tau_{g}=0 . \rho_{n}$ and $\rho_{g}$ are the normal and geodesic curvatures of $C$, respectively, at a point $p \in S$.

Let $\rho$ be the curvature of $\alpha(s)$ at $p$ which is defined by $d \mathbf{T} / d s=\rho \mathbf{N}$, and $\mathbf{N}$ be the principle normal to the curve at $p$. Using the first equation (123) in the Darboux equations (123)-(125) we find

$$
\rho^{2}=\rho_{\mathrm{g}}^{2}+\rho_{\mathrm{n}}^{2} .
$$

Since the tangent vector $\mathbf{T}$ to the curve $C$ is common in both frames it is possible to pass from the Frenet trihedron to the Darboux trihedron by a special local $\mathrm{SO}(3)$ transformation. Let $\mathbf{T}, \mathbf{b}, \mathbf{n}$ define the Darboux trihedron and T, N, B denote the Frenet-Serret triad of orthogonal vectors. Then

$$
\mathbf{n}=\sin \theta \mathbf{N}+\cos \theta \mathbf{B}, \quad \mathbf{b}=\cos \theta \mathbf{N}-\sin \theta \mathbf{B} .
$$

This enables us to connect the torsion $\tau$ and curvature $\rho$ of the curve $C$ to its geodesic torsion $\tau_{g}$, geodesic and normal curvatures $\rho_{g}, \rho_{n}$.

This transformation induces a local SU(2) gauge transformation on the Lax equations (145): Letting $\Phi^{\prime}=S \Phi$, we find

$$
\begin{aligned}
& U^{\prime}=S U S^{-1}+S_{u} S^{-1}, \\
& V^{\prime}=S V S^{-1}+S_{v} S^{-1} .
\end{aligned}
$$

The matrix $S$ is given as

$$
S=\frac{1}{\sqrt{2}}\left(\begin{array}{cc}
e^{-i \theta / 2} & -e^{i \theta / 2} \\
e^{-i \theta / 2} & e^{i \theta / 2}
\end{array}\right) .
$$

In what follows we given an example of how a curve motion can be identified from a given surface.

Proposition 8.1: Consider the surface described in Theorem 2.2 of Ref. 1. This surface is associated with the motion of a curve with curvature and torsion given by

$$
\rho^{2}(s, t)=\left(\frac{U_{2}}{a}\right)^{2}+\left(\frac{U_{3}}{a}\right)^{2},
$$




$$
\tau(s, t)=\frac{U_{1}}{a}-\frac{\left(\frac{U_{2}}{U_{3}}\right)_{s}}{1+\left(\frac{U_{2}}{U_{3}}\right)^{2}},
$$

where $t=v$ and $a=d s / d u$. Here $s$ denotes the arc length. The components $V_{1}, V_{2}$, and $V_{3}$ of the velocity of this curve are defined in terms of $\rho$ and $\tau$ by the differential equations (A1). An orthogonal frame on this curve is

$$
T=\frac{\partial F}{\partial s}=-i \Phi^{-1} \sigma_{1} \Phi, \quad B=-i \Phi^{-1} \sigma_{2} \Phi, \quad N=-i \Phi^{-1} \sigma_{3} \Phi .
$$

Proof: Using $a=d s / d u$ and the definitions of $\Phi$ and $F$ to compute $F_{s s}$ and $N_{s}$, it follows that

$$
\left\langle F_{s s}, N\right\rangle=\frac{U_{2}}{a}, \quad\left\langle N_{s}, B\right\rangle=\frac{U_{1}}{a}, \quad\left\langle F_{s s}, B\right\rangle=-\frac{U_{3}}{a} .
$$

Let $\mathbf{T}, \mathbf{b}, \mathbf{n}$ define the Darboux trihedron associated with the matrices $F_{s}, B, N$ defined in (133). Using the Frenet-Serret equations

$$
\mathbf{T}_{s}=\rho \mathbf{N}, \quad \mathbf{N}_{s}=-\rho \mathbf{T}+\tau \mathbf{B}, \quad \mathbf{B}_{s}=-\tau \mathbf{N},
$$

it follows that

$$
\begin{gathered}
\left\langle F_{s s}, N\right\rangle=\mathbf{T}_{s} \cdot \mathbf{n}=\rho \mathbf{n} \cdot(\sin \theta \mathbf{N}+\cos \theta \mathbf{B})=\rho \sin \theta, \\
\left\langle F_{s s}, B\right\rangle=\mathbf{T}_{s} \cdot \mathbf{b}=\rho \cos \theta \\
\left\langle N_{s}, B\right\rangle=\mathbf{n}_{s} \cdot \mathbf{b}=(\sin \theta \mathbf{N}+\cos \theta \mathbf{B}) \cdot(\cos \theta \mathbf{N}-\sin \theta \mathbf{B}) \\
=\theta_{s}-\tau .
\end{gathered}
$$

Comparing these equations with (A4), we find

$$
\frac{U_{1}}{a}=\theta_{s}-\tau, \quad \frac{U_{2}}{a}=\rho \sin \theta, \quad \frac{U_{3}}{a}=\rho \sin \theta .
$$

Eliminating $\theta$, Eqs. (131) and (132) follow. It is now possible to identify the geodesic curvature $\rho_{g}$, the normal curvature $\rho_{n}$, and the geodesic torsion $\tau_{g}$ of the curve $C$ in terms of the parameters of $S$ :

$$
\begin{gathered}
\rho_{g}=\rho \cos (\theta)=\frac{U_{3}}{a}, \\
\rho_{n}=-\rho \sin (\theta)=\frac{U_{1}}{a}, \\
\tau_{g}=\theta^{\prime}-\tau=\frac{U_{1}}{a} .
\end{gathered}
$$

\section{ACKNOWLEDGMENTS}

We are grateful to F. Finkel for his help with plotting the surfaces. This work is partially supported by TUBITAK, Turkish Academy of Sciences and EPSRC under Grant No. GG/J71885. 


\section{APPENDIX A: THE MOTION OF CURVES}

Let $u$ denote the arclength of a curve in $\mathbb{R}^{3}$. This curve can be uniquely characterized, within a rigid motion in $\mathbb{R}^{3}$, by its curvature and its torsion. This characterization is expressed by the classical Frenet-Serret equations which define the dependence of the associated frame on $u .^{3-9}$

Proposition A.1: Let the scalar real functions $\rho(u, t)$ and $\tau(u, t)$, which are differentiable functions of $u$ and $t$ for every $(u, t)$ in some neighborhood of $\mathbb{R}^{2}$, denote the curvature and torsion of a curve with arclength denoted by $u$. Let the real scalar functions $V_{j}$, which are differentiable functions of $u$ and $t$ for every $(u, t)$ in some neighborhood of $\mathbb{R}^{2}$, denote the velocity of this curve. The motion of this curve is defined by

$$
\frac{\partial \rho}{\partial t}-\frac{\partial V_{1}}{\partial u}+V_{2} \tau=0, \quad \frac{\partial \tau}{\partial t}+\frac{\partial V_{3}}{\partial u}-V_{2} \rho=0, \quad \frac{\partial V_{2}}{\partial u}+V_{1} \tau+V_{3} \rho=0 .
$$

These equations are the compatibility conditions of the following equations for the $\mathrm{SU}(2)$ valued function $\Phi(u, t)$,

$$
\frac{\partial \Phi}{\partial u}=\frac{i}{2}\left(\begin{array}{cc}
\tau & -\rho \\
-\rho & -\tau
\end{array}\right) \Phi, \quad \frac{\partial \Phi}{\partial t}=-\frac{i}{2}\left(\begin{array}{cc}
V_{3} & V_{1}-i V_{2} \\
V_{1}+i V_{2} & -V_{3}
\end{array}\right) \Phi .
$$

Proof: Let $x_{j}, j=1,2,3$, be a point on a curve in $\mathbb{R}^{3}$ whose arclength is denoted by $u$. This leads to

$$
\sum_{j=1}^{3}\left(\frac{\partial x_{j}}{\partial u}\right)^{2}=1
$$

The Serret-Frenet frame is a triad of orthonormal vectors, $\mathbf{T}, \mathbf{N}, \mathbf{B}$, where $\mathbf{T}$ is the tangent vector, $\mathbf{N}$ is the principal normal unit vector, perpendicular to $\mathbf{T}$ which lies in the oscillating plane of the curve, and $\mathbf{B}$ is the binormal unit vector, perpendicular to both $\mathbf{T}$ and $\mathbf{N}$. The components of these vectors satisfy the condition

$$
T_{j}^{2}+N_{j}^{2}+B_{j}^{2}=1, \quad j=1,2,3,
$$

and the classical Frenet-Serret equations

$$
\frac{\partial}{\partial u}\left(\begin{array}{c}
T_{j} \\
N_{j} \\
B_{j}
\end{array}\right)=\left(\begin{array}{ccc}
0 & \rho & 0 \\
-\rho & 0 & -\tau \\
0 & \tau & 0
\end{array}\right)\left(\begin{array}{c}
T_{j} \\
N_{j} \\
B_{j}
\end{array}\right), \quad j=1,2,3 .
$$

Suppose that the above curve is allowed to evolve in time and that it does not stretch during the motion. Since the frame is orthogonal, its time evolution is given by

$$
\frac{\partial}{\partial t}\left(\begin{array}{c}
T_{j} \\
N_{j} \\
B_{j}
\end{array}\right)=\left(\begin{array}{ccc}
0 & V_{1} & -V_{2} \\
-V_{1} & 0 & V_{3} \\
V_{2} & -V_{3} & 0
\end{array}\right)\left(\begin{array}{c}
T_{j} \\
N_{j} \\
B_{j}
\end{array}\right), \quad j=1,2,3 .
$$

Using the $\operatorname{su}(2)$ representation of so(3), these equations yield (A2).

Proposition A.2: Let the complex valued functions $\psi(u, t, \lambda)$ and $V(u, t, \lambda)$, be differentiable functions of $u$ and $t$ for every $(u, t)$ in some neighborhood of $\mathbb{R}^{2}$. Assume that $\psi$ and $V$ satisfy

$$
\psi_{t}=V_{u}+i \sigma \psi-i \lambda V
$$

where $\sigma$ is defined by 


$$
\sigma_{u}=\frac{-i}{2}(\bar{V} \psi+V \bar{\psi})
$$

Equations (A6) and (A7) are the compatibility conditions of the following equations for the $\mathrm{SU}(2)$ valued function $\Phi(u, t, \lambda)$ :

$$
\frac{\partial \Phi}{\partial u}=\frac{1}{2}\left(\begin{array}{cc}
i \lambda & \psi \\
-\bar{\psi} & -i \lambda
\end{array}\right) \Phi, \quad \frac{\partial \Phi}{\partial t}=\frac{1}{2}\left(\begin{array}{cc}
i \sigma & V \\
\bar{V} & -i \sigma
\end{array}\right) \Phi .
$$

Equations (A6) and (A7) describe the motion of a curve with $\rho=|\psi|, \tau=(\arg \psi)_{u}+\lambda$. The velocity of this curve satisfies

$$
V_{1}+i V_{2}=V \exp \left[-i \partial_{u}^{-1} \tau-i \lambda u\right], \quad V_{3 u}=-\tau_{t}+\sigma_{u} .
$$

Proof: Substituting the relations

$$
\psi=\rho e^{i \partial_{u}^{-1} \tau+i \lambda u}, \quad V=\left(V_{1}+i V_{2}\right) e^{i \partial_{u}^{-1} \tau+i \lambda u},
$$

into Eqs. (A6) and (A7) we find (A2) and

$$
\partial_{u}^{-1} \tau_{t}=\frac{V_{2 u}+V_{1} \tau}{\rho}+\sigma, \quad \sigma_{u}=V_{2} \rho,
$$

where $\partial_{u}^{-1}$ denotes integration with respect to $u$. Eliminating $\sigma$ from these equations we find that the equation obtained from the equations in (A2) after eliminating $V_{3}$.

Example A.1: (Constant torsion) The motion of a curve of constant torsion $\tau=\lambda$ is characterized by

$$
\rho_{t}=-\lambda V_{2}-\frac{1}{\lambda}\left(\partial_{u}^{2}+\rho^{2}+\rho_{u} \partial_{u}^{-1} \rho\right) V_{2},
$$

where the velocities $V_{1}$ and $V_{3}$ can be expressed in terms of $V_{2}$ and $\rho$ by

$$
V_{1}=-\frac{1}{\lambda}\left(V_{2 u}+\rho \partial_{u}^{-1}\left(V_{2} \rho\right)\right), \quad V_{3}=\partial_{u}^{-1}\left(V_{2} \rho\right) .
$$

Proof: If $\tau=\lambda$ Eq. (A2) becomes

$$
\rho_{\tau}=V_{1 u}-\lambda V_{2}, \quad V_{3 u}=V_{2} \rho, \quad V_{1}=-\frac{1}{\lambda}\left(V_{2 u}+\rho V_{3}\right) .
$$

These equations yield Eq. (A11).

\section{APPENDIX B: INTEGRABLE CURVE MOTIONS}

It is well known that there exist many curve evolutions which are integrable. We call a curve evolution integrable if the motion is defined in terms of an integrable PDE. Integrable evolutions of curves have been studied extensively in the recent literature ${ }^{3-8}$. It turns out that for particular velocities, the motion of curves is defined by certain integrable equations, which include the sine-Gordon, the modified Korteweg-de Vries, the nonlinear Schrödinger, and the Hirota equations. An obvious approach for obtaining integrable curve evolutions is to choose the functions $V_{j}$ in such a way that the nonlinear equations (A1) [or (A6) and (A7)] are independent of $\lambda$.

Example B.1: (Integrable evolutions of curves with constant torsion $\tau$ ) The motion of curve with constant $\tau=\lambda$ is characterized by Eqs. (A11) and (121). Let its velocity be specified as follows: 
Case 1: If

$$
V_{1}=0, \quad V_{2}=-\frac{1}{\lambda} \sin \theta, \quad V_{3}=\frac{1}{\lambda} \cos \theta,
$$

then $\rho$ evolves according to the sine-Gordon equation,

$$
\rho=\theta_{u}, \quad \theta_{u t}=\sin \theta .
$$

Case 2: If

$$
V_{1}=\rho_{u u}+\frac{1}{2} \rho^{3}-\lambda^{2} \rho, \quad V_{2}=-\lambda \rho_{u}, \quad V_{3}=-\frac{1}{2} \lambda \rho^{2}+\lambda^{3},
$$

then $\rho$ evolves according to the modified $\mathrm{KdV}$ equation,

$$
\rho_{t}=\rho_{u u u}+\frac{3}{2} \rho^{2} \rho_{u} .
$$

Case 3: If $\lambda=1$ and

$$
V_{1}=\rho_{u u}+\frac{1}{2} \rho^{3}-\rho, \quad V_{2}=-\rho_{u}, \quad V_{3}=-\frac{1}{2} \rho^{2}+1,
$$

then $\rho$ evolves according to the Painlave II equation

$$
\rho=t^{1 / 3} W(\xi), \quad \xi=u(t)^{-1 / 3}, \quad W_{\xi \xi}+\frac{1}{3} \xi W+\frac{1}{2} W^{3}=C,
$$

where $C$ is a constant.

Example B.2: (Integrable curve evolutions associated with the NLS) Let

$$
V=i \psi_{x}-\lambda \psi, \sigma=\frac{1}{2}|\psi|^{2}+\lambda^{2},
$$

in Eqs. (A7) and (A8), then $\psi$ evolves according to the nonlinear Schrödinger equation,

$$
\psi_{t}=i \psi_{x x}+\frac{i}{2} \psi|\psi|^{2}
$$

This describes the integrable curve motion with $\rho=|\psi|, \tau=\partial_{u} \arg (\psi)+\lambda$.

${ }^{1}$ A. S. Fokas and I. M. Gelfand, "Surfaces on Lie groups, on Lie algebras and their integrability," Commun. Math. Phys. 177, 203 (1996).

${ }^{2}$ A. S. Fokas, L. M. Gelfand, F. Finkel, and Q. M. Liu, "A formula for constructing infinitely many surfaces on lie algebras and integrable equations," (unpublished).

${ }^{3}$ G. L. Lamb, J. Math. Phys. 18, 1654 (1977).

${ }^{4}$ H. Hosimoto, J. Fluid Mech. 51, 477 (1972).

${ }^{5}$ K. Nakayama and M. Wadati, J. Phys. Soc. Jpn. 62, 473 (1993).

${ }^{6}$ M. Gürses, Phys. Lett. A 241, 329 (1998).

${ }^{7}$ R. E. Goldstein and D. M. Petrich, Phys. Rev. Lett. 67, 3203 (1991).

${ }^{8}$ A. Doliwa and P. M. Santini, Phys. Lett. A 185, 373 (1994).

${ }^{9}$ Q. M. Liu, "Generalized conditional symmetries, asymptotic integrability, and integrable surfaces," Ph.D. thesis, Clarkson University, 1995.

${ }^{10}$ M. Gürses and Y. Nutku, "New nonlinear evolution equations from surface theory,”, J. Math. Phys. 22, 1393 (1981).

${ }^{11}$ A. Sym, "Soliton Surfaces," Lett. Nuovo Cimento Soc. Ital. Fis. 33, 394 (1982).

${ }^{12}$ A. Sym, "Soliton Surfaces. II," Lett. Nuovo Cimento Soc. Ital. Fis. 36, 307 (1983).

${ }^{13}$ A. Sym, "Soliton surfaces. III. Solvable nonlinearities with trivial geometry," Lett. Nuovo Cimento Soc. Ital. Fis. 39, 193 (1984); "'Soliton surfaces. IV. Topological charge for 'Nontopological' Solitons," 40, 225 (1984); "Soliton Surfaces. V. Geometric theory of loop solitons," 41, 33 (1984); "Soliton surfaces. VI. Gauge invariance and final formulation of the approach," 41, 353 (1984).

${ }^{14}$ A. Sym, O. Ragnisco, D. Levi, and M. Brushi, "Soliton surfaces, VII. Relativistic string in external field: General integral and particular solutions," Lett. Nuovo Cimento Soc. Ital. Fis. 44, 529 (1985).

${ }^{15}$ A. I. Bobenko, "All constant mean curvature tori in $\mathbb{R}^{3}, S^{3}, H^{3}$ in terms of theta functions," Math. Ann. 290, 209 (1991). 
${ }^{16}$ A. I. Bobenko, "Integrable surfaces" (Russian) Functional Anal. i Prilozhen. 24, $68-69$ (1990); translation in Funct. Anal. Appl. 24, 227-228 (1991); "Surfaces in terms of 2 by 2 matrices, old and new integrable cases," in Harmonic Maps and Integrable Systems, edited by A. P. Fordy and J. C. Wood, Aspects of Mathematics (Friedr. Vieweg and Sohn, 1994), pp. 83.

${ }^{17}$ H. C. Wente, "Twisted tori of constant mean curvature in $\mathbb{R}^{*}$ " in Seminar on New Results in Nonlinear Partial Differential Equations, edited by A. Tromba, Aspects of Mathematics, Vol. E10, (Friedr. Vieweg and Sohn, 1987), pp. $1-36$.

${ }^{18}$ U. Pinkall and I. Sterling, Ann. Math. 130, 407 (1989).

${ }^{19}$ D. A. Korotkin, "On some integrable cases in surface theory," J. Math. Sci. 94, 65 (1999). [Translation of Zapiski Nauchnykh Seminarov Sankt-Petersburgskogo Otdeleniya Matematicheskogo Institute im V. A. Steklova Rossiiskoi Akademii Nauk. 234, (1996)].

${ }^{20}$ J. Tafel, "Surfaces in $\mathbb{R}^{3}$ with prescribed curvature," J. Geom. Phys. 17, 381 (1995).

${ }^{21}$ D. Levi and A. Sym, “'Integrable systems describing surfaces of nonconstant curvature," Phys. Lett. A 149, 381 (1990).

${ }^{22}$ A. Sym, "Soliton surfaces and their applications (soliton geometry from spectral problems)," in Geometrical Aspects of the Einstein Equations and Integrable Systems [Lect. Notes Phys. 239154 (1985)].

${ }^{23}$ G. Konopelchenko, "Induced surfaces and their integrable dynamics,"' Stud. Appl. Math. 96, 9-51 (1996); "Nets in $R^{3}$, their integrable dynamics and the DS hierarchy," Phys. Lett. A 183, 153 (1993).

${ }^{24}$ W. K. Schief, "On the geometry of an integrable $2+1$ dimensional sine-Gordon system," Proc. R. Soc. London, Ser. A 453, 1671 (1997).

${ }^{25}$ J. Cieslinski, P. Goldstein, and A. Sym, "Isothermic surfaces in $E^{3}$ as soliton surfaces," Phys. Lett. A 205, 37-43 (1995).

${ }^{26}$ J. Cieslinski, “A generalized formula for integrable class of surfaces in Lie algebras," J. Math. Phys. 38, 4255 (1997).

${ }^{27}$ T. K. Milnor, "Surfaces in Minkowski 3-space on which $H$ and $K$ are linearly related," Michigan Math. J. 30, 309 (1983).

${ }^{28} \mathrm{H}$. Wu, "Weingarten surfaces and nonlinear partial differential equations," Ann. Global Anal. Geom. 11, 49 (1993).

${ }^{29}$ M. J. Ablowitz and H. Segur, Solitons and the Inverse Scattering Transform (SIAM, 1981).

${ }^{30}$ A. C. Newell, Solitons in Mathematics and Physics (SIAM, 1985).

${ }^{31}$ Important Developments in Soliton Theory, edited by A. S. Fokas and V. Zakharov (Springer, Berlin, 1993).

${ }^{32}$ P. D. Lax, Commun. Pure Appl. Math. 21, 467 (1968).

${ }^{33}$ H. Hopf, Differential Geometry in the Large [Lect. Notes Math. 100 1983].

${ }^{34}$ J. Spruck, "The elliptic sinh-Gordon equation and the construction of toroidal soap bubbles," in Calculus of Variations and Partial Differential Equations, Proceedings, Toronto, 1986 edited by S. Heldebrandt, D. Kinderlehrer, M. Miranda [Lect. Notes Math. 1340273 1988].

${ }^{35}$ H. C. Wente, Pac. J. Math. 121, 193 (1986).

${ }^{36}$ U. Abresch, J. Reine Angew. Math. 394, 169 (1987).

${ }^{37}$ R. Walter, Geom. Dedicata 23, 187 (1987).

${ }^{38}$ P. J. Olver, Applications of Lie Groups to Differential Equations (Springer, Berlin, 1991).

${ }^{39}$ I. M. Gel'fand and L. A. Dikii, Usp. Mat. Nauk 30, 67 (1975); A. S. Fokas, Stud. Appl. Math. 77, 253 (1987).

${ }^{40}$ M. P. Do Carmo, Differential Geometry of Curves and Surfaces (Prentice-Hall, Englewood Cliffs, NJ, 1976), p. 261.

${ }^{41}$ L. P. Eisenhart, Transformation of Surfaces, 2nd edition (Chelsea, New York, 1962). 